\section{Yoghurt from short supply chain: preliminary study of microbiological and physicochemical characteristics during shelf life}

\author{
Virginia Carfora, ${ }^{1}$ Nicla Marri, ${ }^{1}$ \\ Carmela Tripaldi, ${ }^{2}$ Daniela Patriarca, ${ }^{1}$ \\ Giuseppina Giacinti, ${ }^{1}$ Carlo Boselli, ${ }^{1}$ \\ Gilberto Giangolini, ${ }^{1}$ Simonetta Amatiste ${ }^{1}$ \\ 'Istituto Zooprofilattico Sperimentale \\ delle Regioni Lazio e Toscana, Centro di \\ Referenza Nazionale per la qualità del \\ latte e dei derviati ovini e caprini, Roma; \\ ${ }^{2}$ Centro di Ricerca per la produzione delle \\ carni e il miglioramento genetico \\ (Roma-Tormancina) (CRA-PCM), \\ Monterotondo, Italy
}

\section{Abstract}

Yoghurt is one of very popular flavorful and healthful dairy product obtained by fermentation of lactic acid bacteria including Lactobacillus delbrueckii bulgaricus and Streptococcus thermophilus. Its production and consumption is growing continuously due to its therapeutic properties beside its high nutritive value. Thirty samples of yoghurt from short supply chain produced in 2 factory localized in Lazio region were analyzed with the aim of determining how certain microbiological and physicochemical characteristics change during their shelf life. Different types of yoghurt were studied: plain (12), fruit (14) and cereal yoghurt (4) produced with cow (8) and goat milk (22).The obtained results show: no presence of Enterobacteria, occasional presence of moulds and a considerable presence of yeasts. On the other hand, all the products analyzed have shown an almost constant amount of lactic acid bacteria during their shelf life. Lactic acid bacteria were identified by a biochemical and polymerase chain reaction assay. The presence of undesired microorganisms like yeasts was found. However, the quality of products was satisfying for the concentration of lactic acid bacteria detected in their shelf life.

\section{Introduzione}

Le aziende da latte negli ultimi tempi cercano nella trasformazione diretta una opportunità per ampliare e diversificare la commercializzazione dei loro prodotti, anche sulla spinta di una forte richiesta da parte dei consumatori. Si riscontra un incremento della trasforma- zione in azienda soprattutto negli allevamenti di piccoli ruminanti dato l'apprezzamento dei prodotti diversi da quelli a base di latte bovino da parte di una nutrita schiera di consumatori.

Le maggiori differenze nella composizione del latte caprino e di quello bovino sono soprattutto legate alla diversa proporzione e caratteristiche chimiche delle caseine presenti, come anche alla struttura e alle dimensioni dei globuli di grasso e delle micelle proteiche. Tali aspetti sono direttamente coinvolti nella trasformazione della matrice latte durante il processo di produzione e di conseguenza influenzano la qualità dei prodotti finiti. Il latte caprino è caratterizzato da un elevato valore nutrizionale legato in particolar modo alla maggiore digeribilità della componente proteica e lipidica (Eissa et al., 2010; Dave et al., 1997). A tal proposito, è opportuno ricordare l'importanza del latte caprino, dei formaggi e dello yogurt nell'alimentazione umana: questi prodotti sono frequentemente impiegati nel trattamento di fenomeni allergici e disordini gastrointestinali legati al consumo di latte bovino (Birollo et al., 2000).

Lo yogurt è il prodotto ottenuto per coagulazione acida del latte senza successiva sottrazione di siero per azione esclusiva dell'azione di due microrganismi specifici in associazione: Lactobacillus delbrueckii bulgaricus e Streptococcus thermophilus (Chandan, 2006). La tecnologia di produzione dello yogurt prevede l'esecuzione delle seguenti operazioni: standardizzazione del latte con particolare riferimento al titolo di grasso e di residuo secco magro; omogeneizzazione per la stabilizzazione fisica dei globuli di grasso; pastorizzazione ad alta temperatura per eliminare eventuali microrganismi patogeni presenti e per favorire la formazione di complessi proteici con elevata capacità di trattenere l'acqua da parte del coagulo acido; inoculo con batteri lattici; fermentazione nei serbatoi alla temperatura di $40-45^{\circ} \mathrm{C}$ fino alla formazione del coagulo e al raggiungimento dell'acidità desiderata; raffreddamento e successiva rottura del coagulo. Possono essere aggiunti ingredienti caratterizzanti come frutta, cereali, caffè e altri. Questi vengono di norma addizionati allo yogurt in forma di preparati pastorizzati prodotti da aziende specializzate in confetture e contengono spesso zucchero e altri edulcoranti, aromi, addensanti e stabilizzanti. Si procede quindi con il confezionamento (Bottazzi, 1993).

In assenza di specifiche disposizioni di legge la categoria dei latti fermentati è stata definita in Italia con alcune circolari del Ministero della Sanità (1972, 1974, 1986). Tuttavia alcuni riferimenti (Determinazione Regione Piemonte, 2006) indicano come standard microbiologici l'assenza di microrganismi patogeni, il limite di $10 \mathrm{UFC/g}$ per coliformi e per Staphylococcus aureus, e di 100 UFC/g per
Correspondence: Simonetta Amatiste, Istituto Zooprofilattico Sperimentale delle Regioni Lazio e Toscana, via Appia Nuova 1411, 00178 Roma, Italy.

Tel. +39.067.9099313 - Fax: +39.067 .9099406 .

E-mail: simonetta.amatiste@izslt.it

Key words: Yoghurt, Short supply chain, Goat, Cow, Lactic acid bacteria.

Conflict of interests: the authors declare no potential conflict of interests.

Received for publication: 15 January 2013.

Revision received: 21 February 2013.

Accepted for publication: 22 February 2013.

This work is licensed under a Creative Commons Attribution 3.0 License (by-nc 3.0).

(C) Copyright V. Carfora et al., 2013

Licensee PAGEPress, Italy

Italian Journal of Food Safety 2013; 2:e6

doi:10.4081/ijfs.2013.e6

i miceti. Inoltre i fermenti specifici utilizzati per la produzione dello yogurt devono essere vivi e vitali fino al momento del consumo e in quantità non inferiore a $10^{6} \mathrm{UFC/g}$ (Sangiorgi et al., 2006).

La normale vita commerciale dello yogurt è tra i 20 e i 40 giorni dalla produzione. Rispetto al latte, lo yogurt ha una shelf life più lunga per due ragioni: i) il valore di $\mathrm{pH}$ e ii) il contenuto di flora lattica presente nel prodotto finito. La produzione di acido lattico dovuta alla degradazione degli zuccheri costituisce uno degli effetti desiderabili dell'attività metabolica dei batteri lattici. L'acido lattico prodotto induce nello yogurt un abbassamento del pH fino a valori che oscillano tra 4,4 e 4,0. Tale processo di fermentazione è autolimitante, in quanto i batteri lattici sono sensibili a valori di $\mathrm{pH}$ più bassi. Questa condizione inibisce la crescita di un'ampia varietà di microrganismi patogeni, consentendo un allungamento della vita commerciale dello yogurt (Riazi e Ziar, 2008).

Scopo del presente lavoro è stato quello di analizzare la variazione di alcuni parametri microbiologici e chimico-fisici in campioni di yogurt prodotti in due aziende a filiera corta, durante la shelf life dichiarata dai produttori.

\section{Materiali e Metodi}

Sono stati esaminati 15 yogurt prodotti da due aziende zootecniche da latte: una mista di capre e bovini (Azienda 1) e una solo caprina (Azienda 2), con annesso caseificio aziendale, localizzate nella regione Lazio. In entrambi i casi il latte utilizzato come materia prima è stato prodotto dalla stessa azienda. 
I 15 yogurt sono pervenuti in due unità campionarie per eseguire le prove al tempo 0 (T0) e al tempo 1 (T1), per un totale di 30 campioni. Sono stati analizzati 16 campioni di yogurt provenienti dall'Azienda 1 (8 prodotti con latte caprino e 8 con latte vaccino) con una vita commerciale dichiarata di 10 giorni, e 14 provenienti dall'Azienda 2, tutti prodotti con latte caprino con una shelf life di 30 giorni.

Complessivamente sono state esaminate tre tipologie di yogurt: bianco (12 campioni), alla frutta (14 campioni) e ai cereali (4 campioni). Di questi 8 campioni ottenuti da latte bovino e 22 da latte di capra. Gli yogurt, conservati a $4^{\circ} \mathrm{C}$, sono stati analizzati in due tempi: dopo tre e dieci giorni dalla data di produzione nel caso della Azienda 1; dopo quindici e trenta giorni per i prodotti della Azienda 2. Sono state eseguite le seguenti analisi microbiologiche: numerazione di lieviti e muffe a $25^{\circ} \mathrm{C}$ (ISO, 2004a), numerazione di enterobatteri (ISO, 2004b), numerazione di lattococchi mesofili e lattobacilli (ISO, 2003a), identificazione di Lactobacillus delbrueckii bulgaricus e Streptococcus thermophilus (ISO, 2003b). Lidentificazione dei batteri lattici e dei lieviti è stata eseguita mediante test biochimici miniaturizzati (API CHL, Biomerieux ${ }^{\circledR}$, Marcy l'Etoile, Francia; e API C AUX, Biomerieux ${ }^{\circledR}$ ). Infine le colonie di batteri lattici sono state confermate tramite metodo biomolecolare. Il DNA è stato estratto dalle colonie batteriche cresciute sui terreni specifici mediante bollitura e sottoposto ad analisi in PCR utilizzando i primer e le condizioni di reazione riportati in letteratura per l'identificazione di Strepto coccus thermophilus (Fortina et al., 2003) e Lactobacillus delbrueckii subsp. bulgaricus e subsp. lactis (Torriani et al., 1999). Gli amplificati, ottenuti tramite l'utilizzo di GeneAmpâ PCR System 9700 (Technologies Ltd, Paisley, UK), sono stati esaminati tramite corsa elettroforetica su gel di agarosio al 1,2\% contenente Gel Red ${ }^{\circledR}$ (Biotium Inc., Hayward, CA, USA) in tampone Tris-acetato-EDTA $1 \mathrm{X}$ e visualizzati con transilluminatore.

Solo sui campioni della Azienda 2 è stato determinato il valore di $\mathrm{pH}$ mediante $\mathrm{pH}$-metro MP225 (Mettler-Toledo, Greifensee, Svizzera) con sonda per la rilevazione della temperatura ed elettrodo combinato: raggiunta la temperatura di $25^{\circ} \mathrm{C}$ gli yogurt sono stati sottoposti alla determinazione del $\mathrm{pH}$ ripetendo in doppio le misurazioni per ogni campione.

\section{Risultati}

Fino al termine della shelf life dichiarata, i prodotti esaminati hanno mantenuto una concentrazione di batteri lattici pressoché costante (Figure 1 e 2). La presenza di lieviti è stata rilevata nei prodotti provenienti dall'Azienda 1 solo

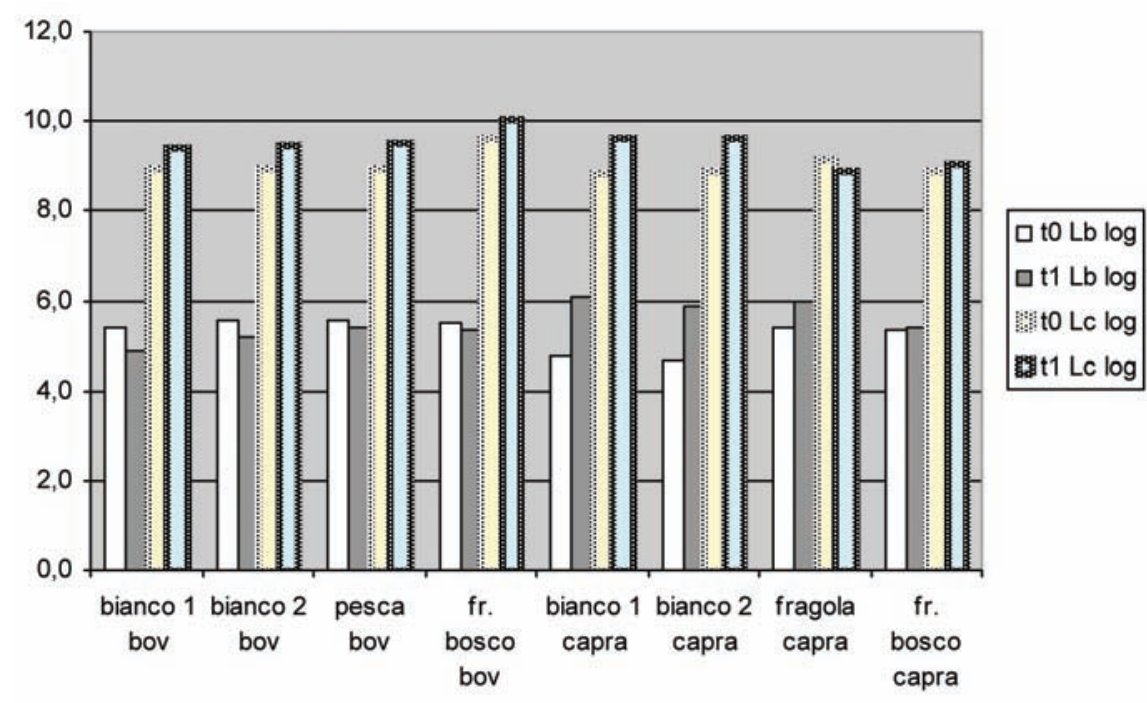

Figura 1. Campioni Azienda 1: concentrazione (UFC/mL) di Lattobacilli (Lb) e Lattococchi (Lc) in yogurt bovino (bov) e di capra al tempo 0 (t0) e al tempo 1 (t1).

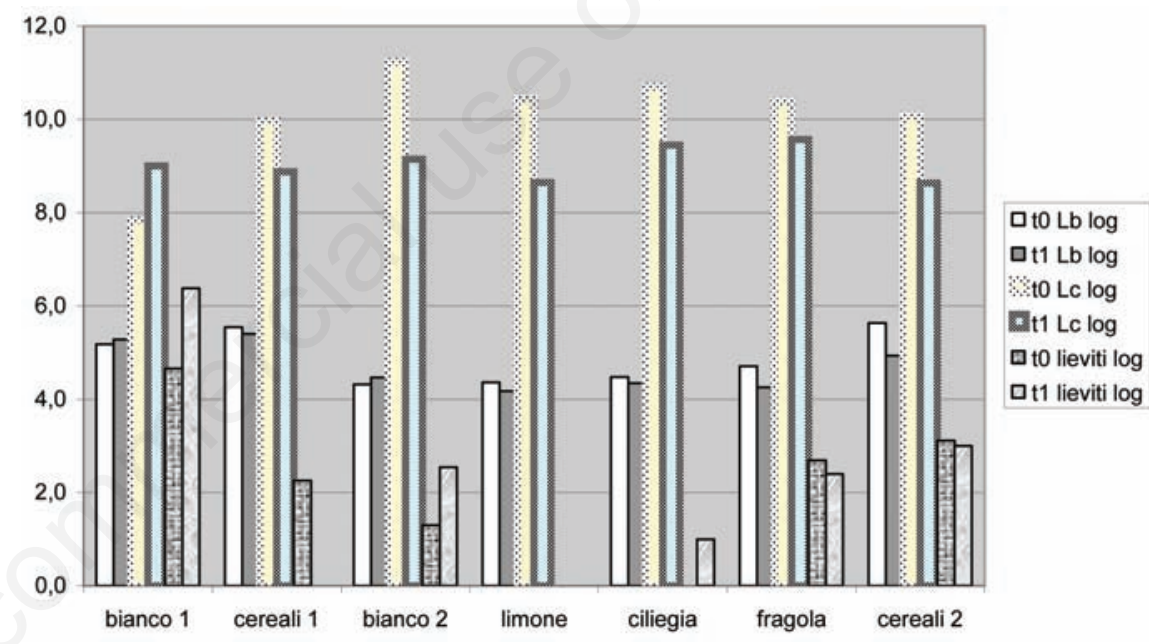

Figura 2. Campioni Azienda 2: concentrazione (UFC/mL) di Lattobacilli (Lb), lattococchi (Lc) e lieviti in yogurt di capra al tempo 0 (t0) e al tempo 1 (t1).

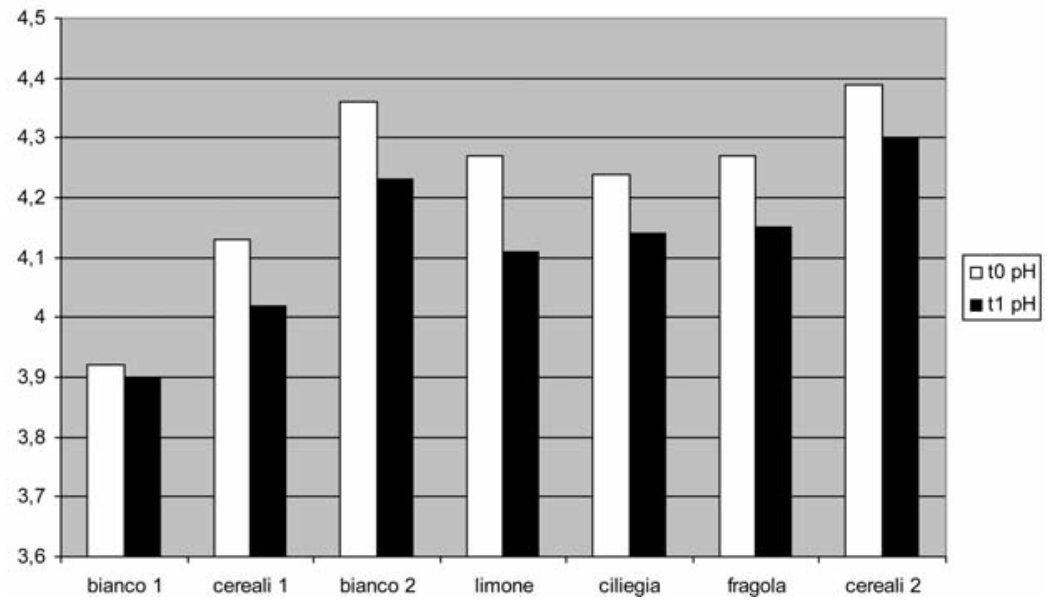

Figura 3. Campioni Azienda 2: valori di $\mathrm{pH}$ in yogurt di capra al tempo 0 (t0) e al tempo $1(\mathbf{t} 1)$. 
negli yogurt alla frutta ed è risultata inferiore a $10 \mathrm{UFC} / \mathrm{mL}$. Negli yogurt dell'Azienda 2 è stata rilevata la presenza di lieviti sia a $\mathrm{T} 0$ che a $\mathrm{T} 1$ in concentrazioni maggiori da 2,0 a $2,4 \times 10^{6} \mathrm{ufc} / \mathrm{mL}$ nelle tipologie di yogurt bianco, cereali e fragola (Figura 2); tale dato può essere probabilmente dovuto a una contaminazione del prodotto durante il confezionamento. La tipizzazione dei lieviti isolati dai campioni dell'Azienda 2 ha rilevato la presenza della sola Candida famata (Senses e Yesim, 2006). È stata rilevata una presenza sporadica di muffe dai campioni esaminati, infatti solo 3 campioni sono risultati positivi su 30 con cariche da 1 a $10 \mathrm{UFC} / \mathrm{mL}$; la concentrazione di enterobatteri rilevata è risultata inferiore a $10 \mathrm{UFC} / \mathrm{mL}$. Il $\mathrm{pH}$ rilevato (Figura 3) corrisponde a quanto riportato in letteratura da 4,0 a 4,36 tranne in un caso dove il $\mathrm{pH}$ è stato di 3,90 . I batteri lattici isolati sono stati identificati con metodi biochimici e confermati mediante metodo biomolecolare: dal confronto dei due metodi è risultata una identificazione sovrapponibile per lo Streptococcus thermophilus. Per il Lactobacillus delbrueckii bulgaricus sono stati ottenuti risultati concordanti solo per i ceppi isolati dai campioni della Azienda 1, mentre per gli isolati provenienti dai campioni della Azienda 2 si è confermata una sola identificazione su 14 (7\%).

\section{Discussione}

Da questa prima indagine si può osservare che gli yogurt prodotti nelle due filiere corte considerate, a fronte di una qualità organolettica interessante (colore, odore, sapore e aspetto) evidenziano all'esame batteriologico la presenza di microrganismi indesiderati quali i lieviti, probabilmente per problemi di contaminazione ambientale (specialmente nei prodotti di una delle due aziende). Tuttavia la qualità dei prodotti risulta essere soddisfacente in merito alla concentrazione di batteri lattici nel corso della shelf life dichiarata. Un altro aspetto interessante emerge dalla parziale discordanza della identificazione dei batteri lattici isolati, segnatamente per il Lactobacillus delbrueckii bulgaricus che ha mostrato una percentuale di conferma molto bassa sui ceppi provenienti dallo yogurt della Azienda 2.

\section{Conclusioni}

È senz'altro fondamentale proseguire nello studio di questi yogurt prodotti nella filiera corta per due ordini di motivi: la necessità di fornire ai consumatori dei prodotti sicuri dal punto di vista microbiologico oltre che organolettico e la possibilità da parte dei produttori di essere consapevoli della reale shelf life degli stessi.

\section{Bibliografia}

Birollo GA, Reinheimer JA, Dinderola CJ, 2000. Viability of lactic acid microflora in different types of yoghurt. Food Res Int 33:799805.

Bottazzi V, 1993. Microbiologia e biotecnologia lattiero-casearia. Il Sole 24 Ore Edagricole, Bologna, Italia.

Chandan RC, 0'Rell KR, 2006. Ingredients for yogurt manufacture. In: Chandan RC, ed. Manufacturing Yoghurt and fermented milks. Wiley-Blackwell Publishing, Hoboke, NJ, pp 179-93.

Dave RI, Shah NP, 1997. Viability of yoghurt and probiotic bacteria in yoghurt made from commercial starter cultures. Int Dairy J 7:31-41.

Determinazione Regione Piemonte, 2006. Criteri microbiologici per prodotti alimentari non soggetti a specifica normativa di legge. Determinazione n. 100/2006 - All. 2 rev.02/2006. Regione Piemonte ed., Torino, Italia.

Eissa EA, Ahmed Mohamed IA, Yagoub AEA, Babiker EE, 2010. Physicochemical, microbiological and sensory characteristics of yoghurt produced from goat milk. Livest Res Rural Dev 22:137. Disponibile al sito: http://www.Irrd.org/lrrd22/8/eiss22137.htm

Fortina GM, Ricci G, Acquati A, Zeppa G, Gandini A, Manichini PL, 2003. Genetic characterization of some lactic acid bacteria occurring in an artisanal protected denomination origin (PD0) Italian cheese, the Toma piemontese. Food Microbiol 20:397-404.

ISO, 2003a. Yoghurt. Enumeration of characteristic microorganisms. Colony-count technique at 37 degrees ${ }^{\circ} \mathrm{C}$. Norma ISO 7889:2003 (IDF 117:2003). Organizzazione internazionale per la normazione ed., Ginevra, Svizzera.

ISO, 2003b. Yoghurt. Identification of characteristic microorganisms (Lactobacillus delbrueckii subsp. bulgaricus and Streptococcus thermophilus). Norma ISO 9232:2003 (IDF 146:2003). Organizzazione internazionale per la normazione ed., Ginevra, Svizzera.

ISO, 2004a. Milk and milk products. Enumeration of colony-forming units of yeasts and/or moulds. Colony-count technique at 25 degrees ${ }^{\circ} \mathrm{C}$. Norma ISO 6611:2004 (IDF 94:2004). Organizzazione internazionale per la normazione ed., Ginevra, Svizzera.

ISO, 2004b. Microbiology of food and animal feeding stuffs. Horizontal methods for the detection and enumeration of Enterobacteriaceae. Part 2: Colony-count method. Norma ISO 21528-2:2004. Organizzazione internazionale per la normazione ed., Ginevra, Svizzera.

Ministero della Sanità, 1972. Produzione e commercio dello yoghurt. Circolare no. 2 del 4 gennaio 1972. Ministero della Sanità ed., Roma, Italia.

Ministero della Sanità, 1974. Produzione e commercio delle preparazioni lattee speciali. Circolare no. 40 del 12 marzo 1974. Ministero della Sanità ed., Roma, Italia.

Ministero della Sanità, 1986. Preparazioni lattee speciali (art. 46 del R.D. 9 maggio 1929 , n. 994). Modificazioni ed integrazioni alle circolari n. 40 del 12 marzo 1974, e n. 2 del 4 gennaio 1972. Circolare no. 9 del 3 febbraio 1986. Ministero della Sanità ed., Roma, Italia.

0'Rell KR, Chandan RC, 2006. Yogurt: fruit preparations and flavoring materials. In: Chandan RC, ed. Manufacturing Yoghurt and fermented milks. Wiley-Blackwell Publishing, Hoboke, NJ, pp 151-60.

Riazi A, Ziar H, 2008. Growth and viability of Yoghurt starter organisms in honey-sweetened skimmed milk. Afr J Biotechnol 7:2055-63.

Sangiorgi F, Aureli F, Boniglia C, Pellegrino LM, Agostoni CV, De Noni I, Resmini P, Zecconi A, Capelletti M, Catillo G, 2006. Libro bianco sul latte e sui prodotti lattiero-caseari. Analisi delle conoscenze scientifiche e considerazioni sul consumo di latte derivati. Assolatte, Milano, Italia.

Senses S, Yesim Z, 2006. Characterization of the yeast flora present in some Turkish high-sugar products. J Gen Appl Microbiol 52:99-106.

Torriani S, Zapparoli G, Dellaglio F, 1999. Use of PCR-Based Methods for Rapid Differentiation of Lactobacillus delbrueckii subsp. bulgaricus and L. delbrueckii subsp. lactis. Appl Environ Microb 65:4351-6. 